ИЗВЕСТИЯ АКАДЕМИИ НАУК ЭСТОНСКОИ ССР. ФИЗИКА МАТЕМАТИКА PROCEEDINGS OF THE ACADEMY OF SCIENCES OF THE ESTONIAN SSR. PHYSICS * MATHEMATICS

$1985,34,1$

\title{
ИССЛЕДОВАНИЕ СОСТАВЛЯЮЩИХ СПЕКТРА ВЫХОДНОГО НАПРЯЖЕНИЯ СИСТЕМЫ ТИРИСТОРНЫХ ВЫПРЯМИТЕЛЕЙ С ОБЩЕЙ ВЕНТИЛЬНОЙ ГРУППОЙ
}

\author{
(Представил Н. Эпик)
}

\section{Введение}

При исследовании выходного напряжения системы выпрямителей, которая состоит из автономных тиристорных регуляторов и общей вентильной группы $\left[{ }^{1}\right]$, возникла необходимость анализа спектра относительно сложных кусочно-синусоидальных сигналов. Поскольку указанные выпрямители дополняют известное множество мостовых выпрямителей, оказалось целесообразным аналитическое описание спектра всех мостовых выпрямителей по единой «цепной» методике, несмотря на то что, например, описание 6-пульсных выпрямителей очень сжатое и обобщенное $\left[{ }^{2}\right]$. Исследовались составляющие спектра низких порядков (150 и 300 Гц), которые необходимо знать при расчете фильтра нижних частот, т. к. составляющие спектра высоких частот не представляют интереса с точки зрения практики. Однако по отмеченным частотам (для сети 50 Гц) невозможно четко определить, которая из них первая, вторая или субгармоническая (ниже будет показано, что из-за реальной общей части всегда присутствует составляющая 150 Гц, однако ее амплитуда может быть незначительной). При анализе приняты следующие упрощающие допущения: вентили рассматриваются в виде идеальных ключей, сеть предполагается симметричной, угол управления $\alpha \leqslant \pi / 3$ (что соответствует работе в энергетически выгодном диапазоне регулирования), напряжение сети синусоидальное, т. е. при анализе мы пренебрегаем коммутационными помехами соседних тиристорных регуляторов. Коммутационные процессы учитывались в случае «реальных» выпрямителей. Все аналитические выражения «идеальных» выпрямителей получены как вырожденные варианты «реальных» выпрямителей при нулевом угле коммутации $\gamma=0$. Тем не менее аналитические выражения «идеальных» выпрямителей представляют практический интерес, поскольку достаточно хорошо описывают физическое содержание электромагнитных процессов и в некоторых случаях пригодны для инженерных расчетов.

При исследовании мы пользовались линейностью преобразования Фурье, которое позволяет разложить сложный сигнал на сигнал с известным спектром и дополняющий сигнал, изученный спектр которого суммируется со спектром известного сигнала $\left[{ }^{3}\right]$. Эта методика позволяет произвести цепной анализ спектра от простого случая к сложному. Основные результаты анализа представлены в относительных единицах в табличной форме, что позволяет сократить объем статьи без потери наглядности; амплитуды гармоник представлены в отношении амплитуды линейного напряжения на входе моста аналогично $\left[{ }^{2}\right]$. 

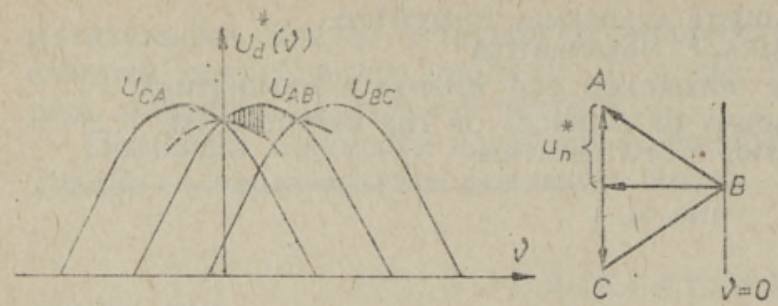

Рис. 1. Векторы, участвующие в формировании выходного напряжения выпрямителя.

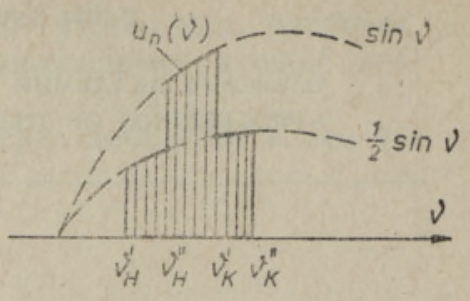

Рнс. 2. Эпюра обобщенной помехн.

На рис. 1 показаны фазовые соотношения напряжений, участвующих в образовании выходного напряжения выпрямителя $u_{d}{ }^{*}(\vartheta)$. Вне коммутационного интервала $\vartheta \notin \gamma$ к нагрузке приложено одно из линейных напряжений $U_{d} \in\left\{U_{C A}, U_{A B}, U_{B C} \ldots\right\}$. Если совместить начало оси абсцисс с точкой естественной коммутации, то

$$
u_{d}^{*}(\vartheta)=\sin (\vartheta+\pi / 3)
$$

В коммутационном интервале $\vartheta \in \mathcal{Y}$ напряжение на нагрузке описывается опережающим вектором

$$
u_{d}^{*}(\vartheta)=(\sqrt{3} / 2) \sin (\vartheta+\pi / 3) \mathrm{e}^{-j \pi / 3} .
$$

Дополняющий сигнал (для простоты в дальнейшем - сигнал помехи)

$$
u_{\text {II }}^{*}(\vartheta)=(\sin \vartheta) / 2,
$$

на рис. 1 показан штриховкой. Однако помеха возникает не только в ходе коммутации, но и в ходе отпирания тиристора в связи с запаздыванием последнего (напр., относительно известного опережающего тиристора и т. п.). Поэтому на рис. 2 приведена форма обобщенной помехи

$$
\begin{array}{lll}
u_{\mathrm{I}}^{*}(\vartheta)=(\sin \vartheta) / 2 & \text { при } & \vartheta_{\mathrm{H}}^{\prime}<\vartheta<\vartheta_{\mathrm{H}}^{\prime \prime}, \\
u_{\mathrm{I}}^{*}(\vartheta)=(\sin \vartheta) / 2 \pm(\sin \vartheta) / 2 & \text { при } & \vartheta_{\mathrm{H}}^{\prime \prime}<\vartheta<\vartheta_{\mathrm{K}}^{\prime}, \\
u_{\mathrm{II}}^{*}(\vartheta)=(\sin \vartheta) / 2 & \text { при } & \vartheta_{\mathrm{K}}^{\prime}<\vartheta<\vartheta_{\mathrm{K}}^{\prime \prime} .
\end{array}
$$

Конкретные значения $\vartheta_{H}^{\prime}, \vartheta_{H}^{\prime \prime}, \vartheta_{\kappa}^{\prime \prime}$ и $\vartheta_{\mathrm{K}}^{\prime \prime}$ указаны ниже. При нахождении коэффициентов Фурье помехи можем осуществить эквивалентное преобразование

$$
\begin{gathered}
c=C(v) \int_{\vartheta_{\mathrm{u}}^{\prime}}^{\theta_{\mathrm{I}}^{\prime \prime}} u_{\mathrm{II}}^{*}(\vartheta) \cdot f(v \vartheta) d \vartheta= \\
=C(v) \int_{\vartheta_{\mathrm{H}}^{\prime}}^{\theta_{\mathrm{K}}^{\prime \prime}} u_{\mathrm{II}}^{*}(\vartheta) f(v \vartheta) d \vartheta+C(v) \int_{\theta_{\mathrm{H}}^{\prime \prime}}^{\vartheta_{\mathrm{K}}^{\prime \prime}} u_{\mathrm{II}}^{*}(\vartheta) f(v \vartheta) d \vartheta ;
\end{gathered}
$$

где $c \in\{a, b\}-$ искомый коэффициент Фурье,

$C(v)$ - константа, зависящая от порядка гармоник,

$$
f(v \vartheta) \in\{\sin v \vartheta, \cos v \vartheta\},
$$

$v-$ номер порядка гармонической составляющей.

Другими словами, характер обобщенной помехи позволяет один слож- 
ный сигнал интегрировать как сумму двух перекрывающихся простых сигналов, одинаковых по структуре. Соответственно исходные формулы коэффициентов Фурье всех рассмотренных выпрямителей имеют одинаковую структуру и отличаются лишь по значениям и по числу членов. Однако это обстоятельство не полностью отражается в табл. $1-6$, так как в них приводятся формулы, преобразованные к виду, удобному для программирования. Формулы для амплитуды составляющих $\rho_{v}$ приведены лишь для несложных простых выпрямителей: сводные формулы недостаточно наглядны и непригодны для программирования при цифровом анализе $\left[{ }^{4}\right]$.

\section{Гармонические составляющие диодного мостового выпрямителя}

При исследовании идеализированного выпрямителя (табл. 1) отсутствуют помеха $\gamma=0$ и составляющая 150 Гц. Поскольку переменная составляющая выходного напряжения - четная функция, то она содержит лишь косинусный член ряда Фурье. Для составляющей 300 Гц $v=1$ можно найти

$$
\begin{gathered}
a_{300}=(6 / \pi) \int_{0}^{\pi / 3} \sin (\vartheta+\pi / 3) \cos 6 v \vartheta d \vartheta=(6 / 2 \pi) \int_{0}^{\pi / 3} \sin \vartheta \cos 6 v \vartheta d \vartheta+ \\
+(6 \sqrt{3} / 2 \pi) \int_{0}^{\pi / 3} \cos \vartheta \cos 6 v \vartheta d \vartheta=-(6 / 35 \pi)
\end{gathered}
$$

Таблица 1

Гармонические составляющие выходного напряжения диодного выпрямителя

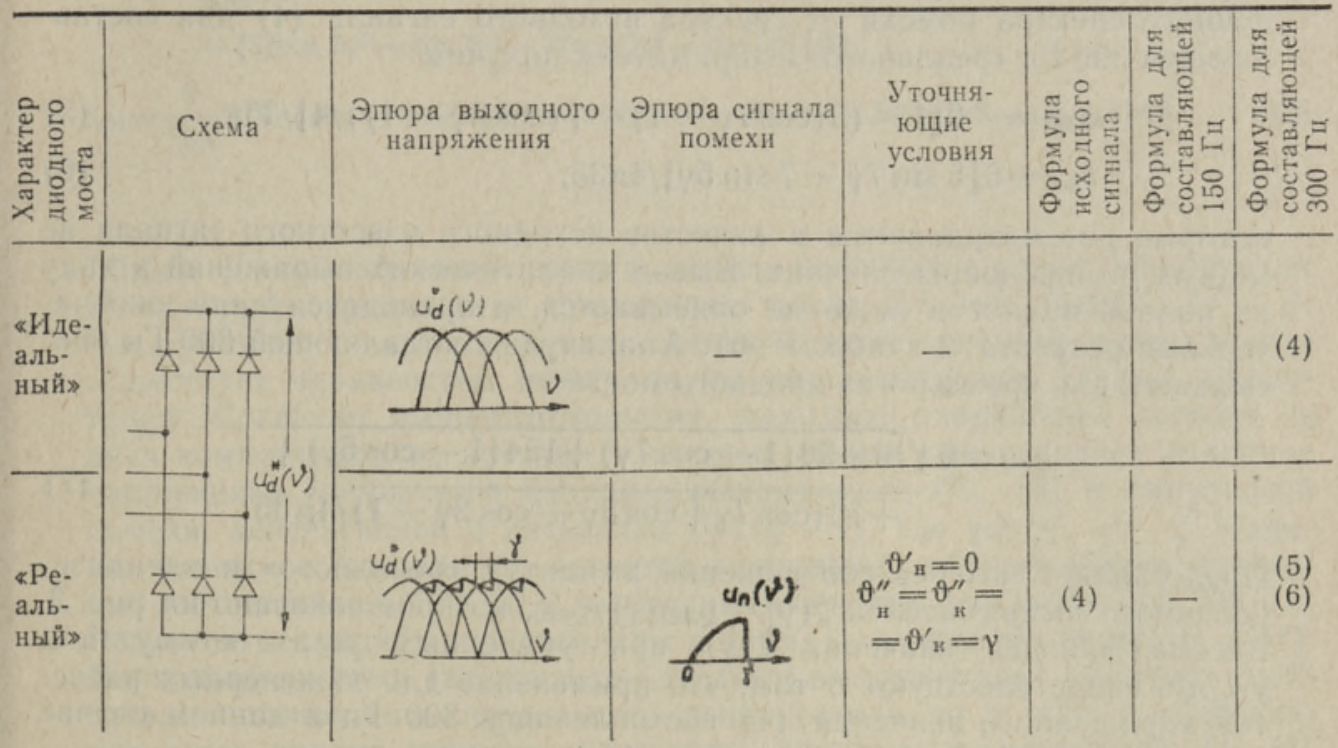

$$
\begin{aligned}
& \left.a_{300}=-\frac{6}{35 \pi}\right] \\
& b_{300}=0 \\
& a_{300}=-\frac{6}{35 \pi}[1-(5(\cos 7 \gamma-1)-7(\cos 5 \gamma-1)) / 4] \\
& b_{300}=\frac{6}{35 \pi}[(5 \sin 7 \gamma-7 \sin 5 \gamma) / 4]
\end{aligned}
$$




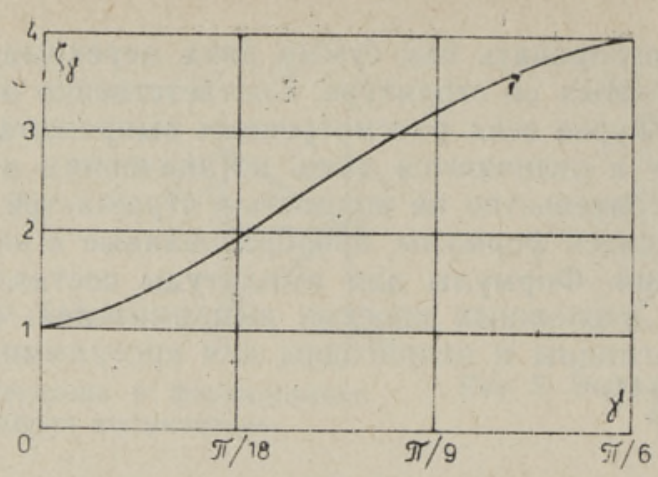

Рис. 3. Относительная пульсация нагруженного неуправляемого мостового выпрямителя.

что соответствует известному из [5] значению. Соответственно амплитуда составляющей 300 Гц следующая

$$
\text { @300 }=6 / 35 \pi .
$$

При исследовании «реального» выпрямителя в интервале $\vartheta \in \gamma$ действует указанная в табл. 1 помеха; найденные по (3) коэффициенты Фурье составляют

$$
\begin{aligned}
& a_{\text {п } 300}=-6[5(\cos 7 \gamma-1)-7(\cos (-5 \gamma)-1)] / 4 \pi 35, \\
& b_{\text {п } 300}=-6[5 \sin 7 \gamma+7 \sin (-5 \gamma)] / 4 \pi 35 .
\end{aligned}
$$

С учетом свойств тригонометрических функций и после вычитания найденного спектра помехи от спектра исходного сигнала (4) для составляющей 300 Гц «реального» выпрямителя получим

$$
\begin{aligned}
& a_{300}=-6[1-(5(\cos 7 \gamma-1)-7(\cos 5 \gamma-1)) / 4] / 35 \pi, \\
& b_{300}=6[5 \sin 7 \gamma-7 \sin 5 \gamma] / 4 \pi 35,
\end{aligned}
$$

которые рассматриваются в качестве исходного известного сигнала во многих последующих случаях. Вывод аналитических выражений в виду их полной аналогии ниже не описывается, а приводятся лишь окончательные результаты (табл. 1-6). Амплитуда составляющей 300 Гц описывается для «реального» диодного моста

$$
\varrho_{300}=6 \sqrt{16+90(1-\cos 7 \gamma)+154(1-\cos 5 \gamma)+}+
$$

Представляет интерес соотношение амплитуд «реального» и идеализированного выпрямителей $\zeta(\gamma)=\varrho_{300}(\gamma) / \varrho_{300}$, которое показано на рис. 3. Весьма большие значения $\zeta(\gamma)$ при умеренных углах коммутации $\gamma<\pi / 6$ свидетельствуют о том, что применение для инженерных расчетов упрощенного значения (4) составляющей 300 Гц в данном случае необосновано.

\section{Гармонические составляющие системы диодных выпрямителей с общей диодной группой}

Свойства системы можно раскрыть на примере уже двух отдельных вентильных групп. Отдельные (напр., катодные) группы диодов работают с углами коммутации $\gamma^{\prime}$ и $\gamma^{\prime \prime}$, определяемыми током нагрузки $I_{d}^{\prime}, I_{d}^{\prime \prime}$. 
Таблица 2

Гармонические составляющие выходного напряжения системы диодных выпрямителей с общей диодной группой

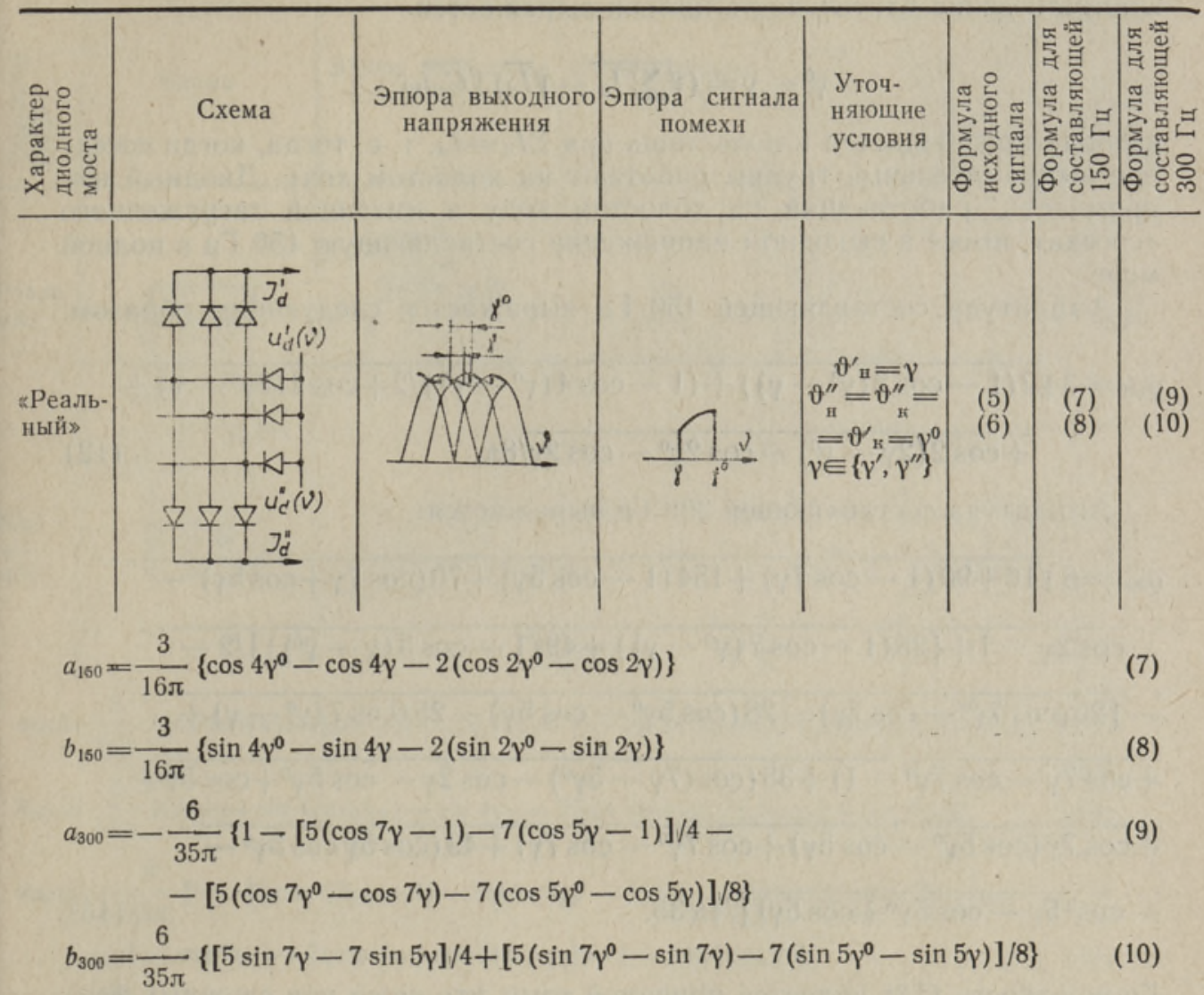

Общая (напр., анодная) группа диодов работает с углом коммутации $\gamma^{0}$, который определяется суммарным током $\Sigma I_{d}>I_{d} I_{d} \in\left\{I_{d}^{\prime}, I_{d}^{\prime \prime} \ldots\right\}$. Следовательно, в выходном напряжении каждой катодной группы всегда существует неравенство между двумя чередующимися углами коммутации. Согласно вышеизложенному, выходное напряжение состоит из двух компонентов, из которых известный сигнал представляет выходное напряжение «реального» диодного выпрямителя $(5),(6)$ и импульсной помехи, действующей в интервале $\vartheta \in\left\{\gamma^{0}-\gamma\right\}$, где $\gamma \in\left\{\gamma^{\prime}, \gamma^{\prime \prime} \ldots\right\}$. Наличие помехи с половинной частотой следования обусловливает появление составляющей 150 Гц и изменения величины составляющей 300 Гц. Результаты анализа представлены в табл. 2, численные значения в [ $\left.{ }^{4}\right]$. Поскольку система «идеальных» диодных выпрямителей обладает свойствами автономного «идеального» выпрямителя, то в табл. 2 представлен лишь анализ системы «реальных» диодных выпрямителей. По $\left[{ }^{6}\right]$ длительность коммутационного интервала выражается зависимостью

$$
t_{\mathrm{K}}=\sqrt{\left[I_{d} L_{s}\left(1+\left(1 / \omega \tau_{1}\right)^{2}\right)\right] / E \omega}=\sqrt{I_{d}} C_{\mathrm{K}},
$$

где $C_{K}-$ постоянная, характеризующая цепь коммутации,

$I_{d}-$ ток нагрузки,

$L_{s}$ - индуктивность цепи коммутации (в основном индуктивность рассеивания трансформатора), 
$\tau_{1}=L_{s} / r_{\partial}-$ постоянная времени цепи коммутации,

$r_{\partial}$ - дифференциальное сопротивление диода,

$E-$ ЕДС в цепи коммутации.

Соответственно длительность помехи выражается

$$
\gamma^{0}-\gamma=\left(\sqrt{\Sigma I_{d}}-\sqrt{I_{d}}\right) \sqrt{C_{\mathrm{K}} / \omega},
$$

которая превращается в ноль лишь при $\Sigma I_{d}=I_{d}$, т. е. тогда, когда соседние выпрямительные группы работают на холостом ходу. Диодный выпрямитель, работающий на холостом ходу и имеющий загруженного «соседа», имеет в выходном напряжении составляющую 150 Гц в полной мере.

Амплитуда составляющей 150 Гц выражается слледующим образом:

$$
Q_{150}=3 \sqrt{2\left(1-\cos 2\left(\gamma^{0}-\gamma\right)\right)+\left(1-\cos 4\left(\gamma^{0}-\gamma\right)\right) / 2+\cos 2\left(2 \gamma^{0}-\gamma\right)+} \cdot
$$

$$
\overline{\mathrm{cos} 2\left(2 \gamma-\gamma^{0}\right)-\cos 2 \gamma^{0}-\cos 2 \gamma} / 8 \pi \text {. }
$$

Амплитуда составляющей 300 Гц выражается:

$\varrho_{300}=6 \sqrt{16+90(1-\cos 7 \gamma)+154(1-\cos 5 \gamma)+70(\cos 7 \gamma+\cos 5 \gamma)-} \cdot$

$-\overline{\cos 2 \gamma-1+[25} \overline{\left.\left(1-\cos 7\left(\gamma^{0}-\gamma\right)\right)+49\left(1-\cos 5\left(\gamma-\gamma^{0}\right)\right)\right] / 2-}$

- ${\overline{\left[20\left(\cos 7 \gamma^{0}-\cos 7 \gamma\right)-28\left(\cos 5 \gamma^{0}-\cos 5 \gamma\right)-25\left(\cos 7\left(\gamma^{0}-\gamma\right)+\right.\right.}}^{\cdot}$



$\left.+\cos 7 \gamma\left(\cos 5 \gamma^{0}-\cos 5 \gamma\right)+\cos 7 \gamma^{0}-\cos 7 \gamma\right)+49\left(\cos 5 \gamma \cos 5 \gamma^{0}-\cdots\right.$

$-\overline{\left.\left.\cos ^{2} 5 \gamma-\cos 5 \gamma^{0}+\cos 5 \gamma\right)\right]} / 4 \pi 35$.

Громоздкость (13) является причиной тому, что ниже при сложных формах сигнала предпочтение отдается табличной форме представления результатов $\left[{ }^{4}\right]$. Контрольный расчет в некоторых точках показал, что рассчитанные по (13) значения амплитуды порядка $10 \%$ выше значений, рассчитанных по (7) без учета влияния общих диодов (напр., $11 \%$ по (13) вместо $10 \%$ по (7)).

\section{Гармонические составляющие несимметричного мостового выпрямителя}

При работе полууправляемого (мостового) выпрямителя (ПУВ) [ $\left.{ }^{7}\right]$ следует учитывать наличие углов коммутации диодов $\gamma_{д}$ и тиристоров $\gamma_{\mathrm{T}}<\gamma_{\text {д }}$, связанных между собой известной $\left[{ }^{5}\right]$ функциональной зависимостью

$$
\gamma_{\mathrm{T}}=\arccos \left[\cos \alpha+\cos \gamma_{\text {д }}-1\right]-\alpha .
$$

Результаты анализа ПУВ приведены в табл. 3, данные расчета по (15)(18) - в [ $\left.{ }^{4}\right]$. Расчеты показывают, что амплитуда составляющей 150 Гц выходного напряжения ПУВ мало зависит от угла коммутации, и следовательно, упрощенные формулы идеального ПУВ (19), (20) могут быть использованы в инженерных расчетах. Амплитуда составляющей 300 Гц, по $\left[{ }^{4}\right]$, существенно зависит от угла коммутации и в этом случае следует воспользоваться уточненными формулами (17), (18). 


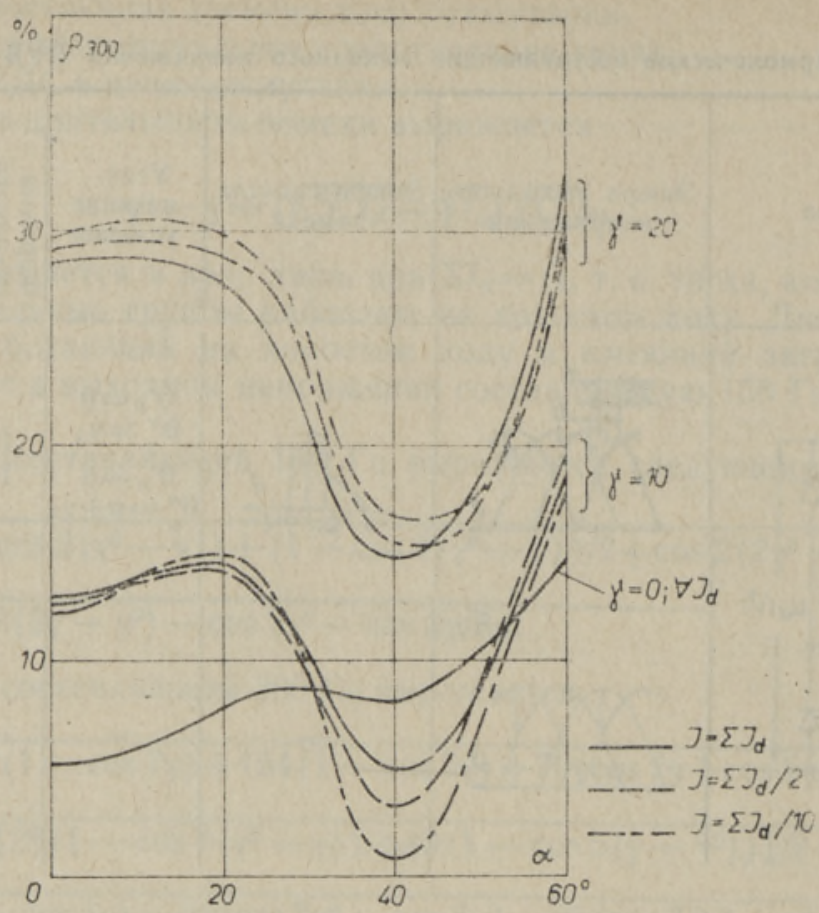

Рис. 4. Составляющая 300 Гц в системе выпрямителей с общей диодной группой.

ная связь между $\gamma_{д}, \gamma_{\mathrm{T}}$. Поскольку угол коммутации общей диодной группы может быть найден по (11), то по (14a) может быть установлен

$$
\gamma_{\mathrm{T}}=\arccos \left[\cos \alpha+\cos \gamma_{\text {д }} \sqrt{I_{d} / \Sigma I_{d}}-1\right]-\alpha,
$$

что свидетельствует о появлении нового фактора, который определяет амплитуду гармонических составляющих. Этот фактор - распределение токов в системе тиристорных регуляторов с общей диодной группой. Случай совместной работы с соседним тиристорным регулятором, работающим на холостом ходу, очевидно, совпадает с таковым автономного «реального» ПУВ. Поэтому в $\left[{ }^{4}\right]$ численные расчеты проведены для случая равномерного $I_{d}=\Sigma I_{d} / 2$ и превосходящего $I_{d}=\Sigma I_{d} / 10$ относительно распределения токов. По указанным расчетам можно заключить, что амплитуда составляющей 150 Гц при малых $\gamma_{д}<\pi / 6$ практически инвариантна в отношении распределения токов между тиристорными регуляторами в системе. Амплитуда составляющей 300 Гц также несущественно зависит от токораспределения, показанного на рис. 4 для $\gamma_{д} \in\{0,10,20\}$. Поэтому в системе тиристорных регуляторов ‘ общей диодной группой для ориентировочных расчетов можно воспользоваться формулами (15) - (22), приняв за основу суммарный ток в общих диодах. Отметим еще, что в системе тиристорных регуляторов с общей диодной группой гармонический состав выходного напряжения опережающего регулятора $\alpha^{\prime}<\alpha^{\prime \prime}$ и запаздывающего регулятора не имеет принципиальных различий.

\section{Гармонические составляющие симметричного (6-пульсного) тиристорного моста}

Схема выпрямителя, эпюры сигналов и помехи, а также расчетные формулы приведены в табл. 4. Несмотря на различающуюся структуру фор- 
Гармонические составляющие симметричного тиристорного мостового выпрямителя

\begin{tabular}{|c|c|c|c|c|c|c|c|}
\hline  & Схема & $\begin{array}{c}\text { Эпюра выходного } \\
\text { напряжения }\end{array}$ & $\begin{array}{c}\text { Эпюра сигнала } \\
\text { помехи }\end{array}$ & $\begin{array}{c}\text { Уточ- } \\
\text { няющие } \\
\text { условия }\end{array}$ & 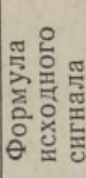 & 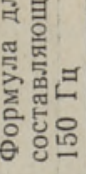 & 造施。 \\
\hline $\begin{array}{l}\text { «Реаль- } \\
\text { ный» }\end{array}$ & & & $\frac{1}{0} v$ & $\begin{array}{c}\theta_{\mathrm{H}}=0 \\
\theta_{\mathrm{H}}^{\prime \prime}=\theta_{\mathrm{K}}^{\prime \prime} \\
\theta_{\mathrm{K}}^{\prime}=a\end{array}$ & $\begin{array}{l}(5) \\
(6)\end{array}$ & - & $\begin{array}{l}(23) \\
(24)\end{array}$ \\
\hline $\begin{array}{l}\text { «иде- } \\
\text { аль: } \\
\text { ный» }\end{array}$ & & & - & - & - & - & $(2$ \\
\hline
\end{tabular}

$a_{300}=\frac{6}{35 \pi}[5(\cos (7 \alpha+\gamma)+\cos 7 \alpha)-7(\cos 5(\alpha+\gamma)+\cos 5 \alpha)] / 4$

$b_{300}=\frac{6}{35 \pi}[5(\sin 7(\alpha+\gamma)+\sin 7 \alpha)-7(\sin 5(\alpha+\gamma)+\sin 5 \alpha)] / 4$

$a_{300}=\frac{6}{35 \pi}[5 \cos 7 \alpha-7 \cos 5 \alpha] / 2$

$b_{300}=\frac{6}{35 \pi}[5 \sin 7 \alpha-7 \sin 5 \alpha] / 2$

мул, результаты их одинаковы $\left[{ }^{2}\right]$. Поскольку этот выпрямитель изучен досканально, нет необходимости останавливаться на его свойствах.

\section{Гармонические составляющие системы тиристорных регуляторов с общей тиристорной группой}

В этой системе между опережающим (табл. 5) и запаздывающим регулятором (табл. 6) есть принципиальная разница. Отличие ведущего (опережающего) регулятора от симметричного моста заключается в том, что общие тиристоры коммутируются сумма́рным током $\Sigma I_{d}>I_{d}^{\prime}$, соответственно увеличивается угол коммутации общих тиристоров $\gamma^{0}>\gamma^{\prime}$. Это обусловливает появление составляющей 150 Гц, которая отсутствует в идеализированной системе. Численные значения амплитуд составляющих 150 и 300 Гц приведены в [ $\left.{ }^{4}\right]$, здесь отметим лишь то обстоятельство, что обе амплитуды есть функции от трех переменных $\varrho=$ $=f\left(\alpha^{\prime}, \alpha^{\prime \prime}, \gamma^{0}, \gamma^{\prime} / \gamma^{0}\right)$, так как $\gamma^{\prime}$ и $\gamma^{0}$ функционально связаны условием (14б). Как и в системе с общей диодной группой, в этой системе расчеты проведены для $I_{d}=\Sigma I_{d} / 2$ и $I_{d}=\Sigma I_{d} / 10$; случай $I_{d}=\Sigma I_{d}$ тождественный таковому автономно симметричного моста. Функции от трех переменных представлены пакетом таблиц. Большой объем данных, иллюстрирующих настоящий анализ, обусловил его представление в виде отдельной статьи $\left[{ }^{4}\right]$. 
Гармонические составляющие ведущего тиристорного регулятора в системе с общей тиристорной группой

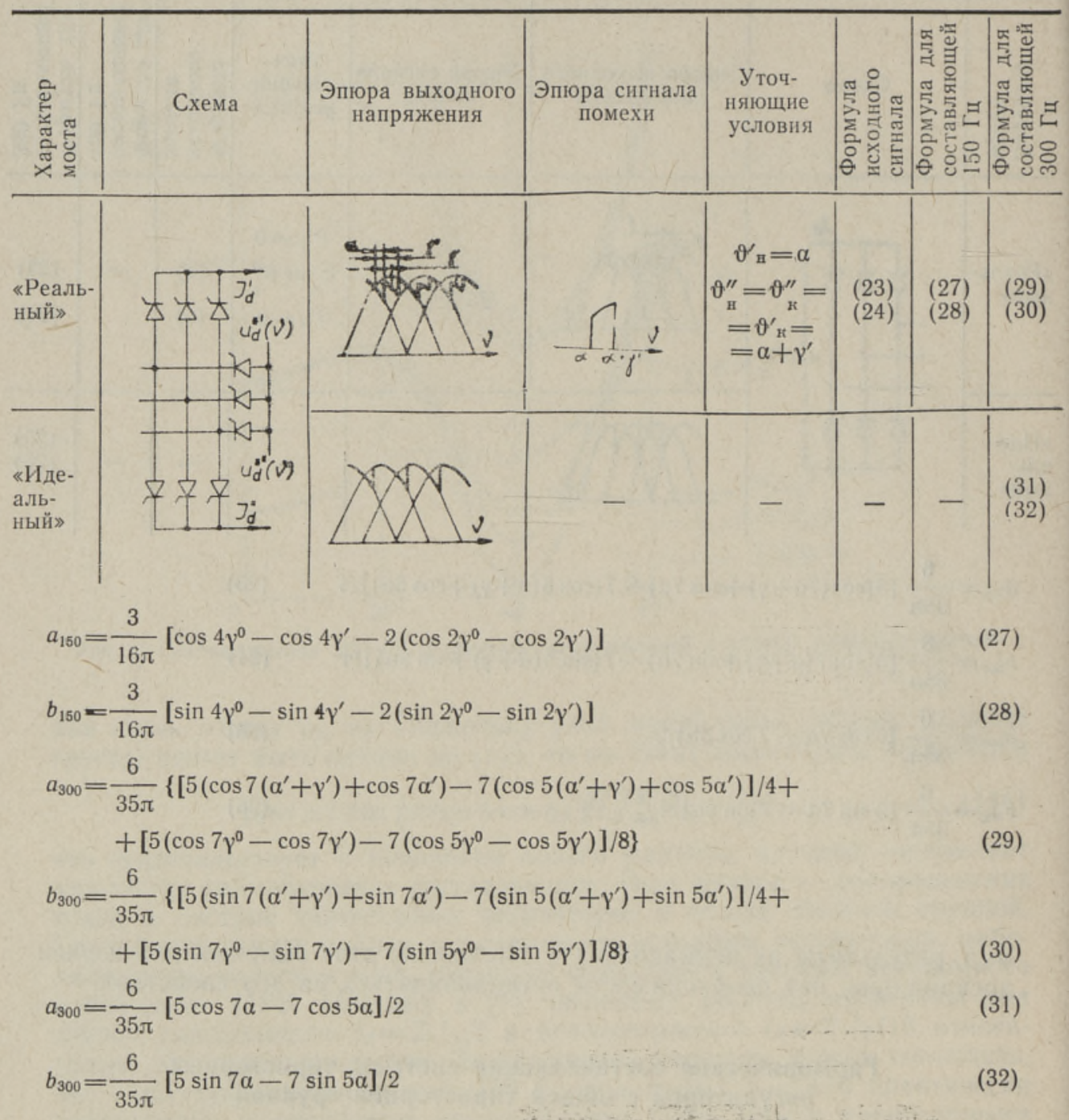

Отличие ведомого (запаздывающего) регулятора от симметричного моста заключается в различии углов управления $\alpha^{\prime \prime}$ и $\alpha^{\prime}$. Последний из них управляет тиристорами общей группы и в общем случае $\alpha^{\prime} \neq \alpha^{\prime \prime}$ всегда (даже для идеальных регуляторов) присутствует составляющая 150 Гц, однако ее значение заведомо меньше, чем в системе тиристорных регуляторов с общей диодной группой. В этом заключается преимущество системы с общими тиристорами перед системой с общими диодами. Остальные положения указаны в табл. 6.

Ввиду многомерности @150 изучен на примере «идеальных» тиристорных регуляторов. Отношение амплитуд составляющих 150 Гц для системы с общей тиристорной группой к амплитуде составляющей 150 Гц для системы с общей диодной группой может быть найдено по (19), (20), (37) и (38) в виде (41) 
Коэффициент подавления $\zeta_{\rho}$ составляющей 150 Гц в системе тиристорных регуляторов с общей тиристорной группой

\begin{tabular}{r|c|c|c|c|c|c|c|c}
\hline \multirow{2}{*}{$\alpha^{\prime \prime}$} & \multicolumn{7}{c}{$\Delta_{\alpha}$} \\
\cline { 2 - 9 } & 0 & 2,5 & 5 & 10 & 15 & 20 & 25 & 30 \\
\hline 5 & 0 & 0,7504 & 1 & & & & & \\
10 & 0 & 0,4398 & 0,7532 & 1 & & & & \\
15 & 0 & 0,3094 & 0,6522 & 0,8943 & 1 & & & \\
20 & 0 & 0,2396 & 0,4472 & 0,7629 & 0,9443 & 1 & & \\
25 & 0 & 0,1966 & 0,3728 & 0,6601 & 0,8580 & 0,9678 & 1 & \\
30 & 0 & 0,1678 & 0,3213 & 0,5827 & 0,7795 & 0,9109 & 0,9808 & 1 \\
40 & 0 & 0,1321 & 0,2563 & 0,4785 & 0,6619 & 0,8040 & 0,9044 & 0,9656 \\
50 & 0 & 0,1117 & 0,2186 & 0,4153 & 0,5859 & 0,7272 & 0,8375 & 0,9167 \\
60 & 0 & 0,0994 & 0,1956 & 0,3764 & 0,5383 & 0,6778 & 0,7928 & 0,8819
\end{tabular}

где $\Delta_{\alpha}=\alpha^{\prime \prime}-\alpha^{\prime}$.

Это соотношение (табл. 7) позволяет дать оценку (сверху) эффекту, получаемому от применения общей тиристорной группы, который увеличивается при глубоком регулировании, т. е. там, где амплитуда составляющей 150 Гц имеет значительную величину.

\section{Выводы}

1. Получены расчетные формулы для гармонических составляющих низких порядков всех простых (некаскадных) мостовых выпрямителей на единой методической основе.

2. Впервые изучены указанные гармонические составляющие для системы тиристорных регуляторов с общими вентильными группами. Показано, что в реальных системах всегда присутствует составляющая 150 Гц.

3. Получена оценка сверху для эффекта подавления составляющей 150 Гц при переходе от общей диодной группы к общей тиристорной группе.

\section{Л И Т Р Р Т У Р А}

1. Тамкиви П., Томсон Т. Изв. АН ЭССР. Физ. Матем., 31, № 4, 454-457 (1982).

2. І линтерник C. Р. Электромагнитные процессы и режимы мощных статических преобразователей. Л., «Наука», 1968.

3. Томсон T. Изв. АН ЭССР. Физ. Матем., 33, № 3, 339-350 (1984).

4. Владимирова О., Томсон Т. Гармонические составляющие низких порядков выходного напряжения системы тирнсторных выпрямителей $є$ общей вентильной группой. Депонирована в ВИНИТИ, № 3410-84.

5. Полупроводниковые выпрямители. (Ред. Ф. И. Ковалев, Г. П. Мостикова). М., «Энергия», 1978.

6. Томсон T. Изв. АН ЭССР. Физ. Матем., 31, № 3, 347-349 (1982).

7. Томсон T., Ерохина Е. Изв. АН ЭССР. Физ. Матем., 32, № 2, 210-217 (1983).

Институт термофизики и электрофизики Академии наук Эстонской ССР
Поступила в редакцию 9/VI 1983 


\section{T. TOMSON}

\section{UHISVENTIILIDEGA ALALDITE SUSTEEMI VALJUNDPINGE SPEKTRI HARMOONILISTEST KOMPONENTIDEST}

Töös on uuritud ühisventiilidega alaldite süsteemi väljundpingete spektraalset koostist $150 \mathrm{~Hz}$ ja $300 \mathrm{~Hz}$ komponentide suhtes, mis on olulised alaldi väljundis rakendatava madalpääsfiltri projekteerimiseks. Kasutades Fourier' teisenduse lineaarsust, on ühtse metoodika alusel leitud kõigi tuntud lihtsildalaldite Fourier' konstantide analüütilised avaldised. Metoodika seisneb uuritava perioodilise signaali lahutamises tuntud perioodiliseks signaaliks ja veasignaaliks, mille leitud spekter summeeritakse tuntud signaali spektriga. Lihtsildalaldite hulga moodustavad dioodsild, dioodsildade süsteem ühisdioodidega; pooljuhitav türistorsild; türistorsildade süsteem ühisdioodidega; türistorsild, türistorsildade süsteem ühistüristoridega. On näidatud, et ülıisventiilide rakendamisega kaasub igas reaalses alaldite süsteemis $150 \mathrm{~Hz}$ komponent. Ideaalalaldi või alaldite süsteem kirjeldub reaalse alaldi kõdunud juhtumina, milles toimub ventiilide hetkeline kommutatsioon.

\section{T. TOMSON}

\section{HARMONIC COMPONENTS IN THE OUTPUT VOLTAGE SPECTRUM OF A RECTIFIER SYSTEM WITH COMMON DEVICE GROUPS}

An investigation of the 150 and $300 \mathrm{~Hz}$ components in the output voltage of rectifier system with common device groups has been carried out. These components are essential for designing low-pass filters used in any rectifier output port. The linearity of the Fourier' transformation gives an opportunity to analyze the spectrum of any unknown signal as a sum of the spectra of the known and error signals. By this method the whole set of non-cascade bridge rectifiers is analyzed by steps. This set of rectifiers consists of the diode bridge, the system of diode bridges with common diodes, the half-controlled thyristor bridge, the system of half-controlled thyristor bridges with common diodes, the thyristor bridge and the system of thyristor bridges with common thyristors. It is shown that in any real system with common devices there exists the $150 \mathrm{~Hz}$ component. An ideal system can be considered as a degenerate of the corresponding real system. 